\title{
Advances and Problems in Preclinical Models for Childhood Cancer
}

\author{
V.Sah
}

SDTITS, India

\section{ABSTRACT}

Microblogging today has gotten an acclaimed specific instrument among Neuroblastoma is a sympathetic nervous system disease in children and is the most prevalent solid tumor in childhood, accounting for $15 \%$ of all pediatric oncology deaths. Nearly $80 \%$ of patients with this clinically active condition do not react to current treatments in the long run. The precise portrayal of tumor biology and diversity is the key obstacle in the discovery and evaluation of novel agents for pediatric drug growth. In addition to this restriction, the low prevalence of neuroblastoma renders it difficult to enroll qualifying patients for early phase clinical trials, emphasizing the importance of thorough preclinical studies to ensure that the right drugs are chosen. To address these issues, researchers need new preclinical models, technologies, and principles. Tissue engineering provides appealing methods for developing threedimensional (3D) cell models utilizing different biomaterials and manufacturing techniques that replicate the geometry, dynamics, heterogeneity, metabolic gradients, and cell connectivity of the native tumor microenvironment. We address existing laboratory models and evaluate their ability to reflect the systemic organization and physiological conditions of the human body, as well as current and emerging strategies to recapitulate the tumor niche utilizing tissue-engineered platforms in this study. Finally, we'll talk about how innovative 3Din vitroculture systems might be used to answer unanswered questions in neuroblastoma biology.
How to cite this paper: V. Sah "Advances and Problems in Preclinical Models for Childhood Cancer" Published in International Journal of Trend in Scientific Research and Development (ijtsrd), ISSN: 2456-6470, Volume-5 | Issue-4, June 2021, pp.762-765, URL: www.ijtsrd.com/papers/ijtsrd42379.pdf

Copyright (C) 2021 by author (s) and International Journal of Trend in Scientific Research and Development Journal. This is an Open Access article distributed under the terms of the Creative Commons Attribution License (CC BY 4.0) (http://creativecommons.org/licenses/by/4.0)

KEYWORDS: Neuroblastoma, Tissue-engineering, childhood cancer

\section{INTRODUCTION}

Neuroblastoma is the most prominent solid tumor in babies [1]. It is a pediatric cancer of the sympathetic nervous system. The prevalence rate of neuroblastoma in children under the age of 15 is 1.2 cases per 100,000 , accounting for around $15 \%$ of all pediatric oncology deaths [2]. Neuroblastoma has a wide range of clinical behavior and effects, varying from complete remission to the emergence of multi-foci- and multi-drug-resistant disorder [1,3]. Nearly $80 \%$ of patients with the clinically active disorder do not react to recent advancements in anticancer therapy, revealing gaps in our knowledge of neuroblastoma biology and our ability to reliably reflect this condition in preclinical models [4,5]. Late therapy toxicities, such as the development of secondary tumors, placed neuroblastoma patients at risk of serious or lifethreatening diseases, emphasizing the need for rigorous and effective preclinical medication screening [6]. Inconsistency between in vitroandinvivotesting results is a major obstacle in drug production for both pediatric and adult cancers, resulting in just 1 in 10 medicines entering clinical trials being accepted by the FDA [7]. Most of the current neuroblastoma research uses two-dimensional (2D) cell culture structures, as well as zebrafish and mouse models. However, since 2D cell culture lacks cellcell and cell-matrix connectivity, metabolic gradients, and cell polarity, it cannot accurately represent the threedimensional (3D) geometry and complexity of human tumors. As a result, current tissue-level awareness of neuroblastoma biology is inadequate to allow confident assumptions regarding patient responses to novel agents that target commonly expressed cellular components (e.g., topoisomerases and microtubules) or widely active signaling pathways (e.g., MAP kinase and AKT). These models also ignore the pediatric background of neuroblastoma, including the immature immune system, medication metabolism discrepancies from adult cancers, and ongoing developmental shifts. Furthermore, the number of children qualifying for early phase clinical trials limits the rigorous assessment of experimental agents in paediatric patients $[5,8]$. Many of these shortcomings had a significant effect on researchers' ability to recognize possible therapeutic targets and prioritize novel agents in the clinical development pipeline due to a lack of expertise and funding. These impediments illustrate potential for researchers to capitalize on the advances achieved in adult cancer science. We may recreate a physiologically important tumor microenvironment in 3Din vitro using correctly sourced and propagated human cells that maintain the fundamental genetic and epigenetic landscape by leveraging developments in tissue engineering and biomaterial growth. Tumors operate at seven different systemic levels inside the human body: chemical, genetic, biochemical, tissue, liver, organ system, and organismal [9]. Each stage shows how intertwined pathways and regulatory feedback loops influence disease development and treatment response. The reconstruction of a reliable physiologically relevant cellular ecosystem becomes progressively difficult as the sophistication of 
structural organization grows, but it is essential for the creation of reliable experimental systems and preclinical models. This study examines the capacity of existing and emerging experimental models to replicate the systemic organization and physiological circumstances of a tumor in a human patient. Finally, we can explore how novel 3Din vitroculture systems may be used to answer several of neuroblastoma biology's unanswered questions.

\section{Tumor environment}

Tumors are known as organs because of their multi-tissue organization and constant exposure to various stimuli and conditions [9]. The tumor micro-environment (TME) is made up of genetic, structural, and molecular components that serve a variety of signaling pathways in the native tumor. The TME is home to a diverse cellular community of cancerous and noncancerous cells such as fibroblasts, stromal cells, and immune cells that move from the bloodstream and adjacent tissues. Secreted factors and extracellular matrix (ECM) proteins, which shape basic 3D physical scaffolding, characterize the TME. At the tissue and organ stages, both cellular and molecular components form TME architecture and sustain its homeostasis. The function of the tumor microenvironment (TME) in disease development, patient prognosis, and therapeutic response is well understood [reviewed elsewhere [10, 11]]. Understanding tumor activity at the tissue level requires differentiating the relative roles of these cellular, molecular, and micro environmental mechanisms in disease progression. The reconstruction of a TM in vitro, where cellular and molecular components of the system can be inter-changeable and finely regulated utilizing tissue-engineered strategies and platforms, can overcome this problem. 2.1. Cellular structure in varying proportions, neuroblastic and Schwann cells make up the neuroblastoma TME. In neuroblastoma, all cell forms have the same progenitor neural crest stem cells, which are found in differing degrees of differentiation. Crosstalk between these neuroblastoma cell types has previously been shown to drive therapy tolerance and Schwann cell proliferation by releasing the growth factor Neuregulin 1 (NRG1) from neuroblastic SH-SY5Y cells [12]. Histo pathological measures such as tumor grade and stromal cell differentiation are used to divide neoplasms into three sub-types-undifferentiated, badly differentiated, and differentiating-and serve as prognostic markers for disease outcome [13]. Depending on the hosting tissue niche, various forms of noncancerous cells can be included in the main and metastatic neuroblastoma microenvironment. Osteoblasts and mesenchymal stem cells are abundant in the bone and bone marrow niches, respectively. Both cell types use paracrine signaling to chemo-attract neuroblastoma cells, which involves many mechanisms working in tandem. They secrete Stromal Cell-Derived Factor-1 (SDF-1), which attaches to chemokine receptors 4 and 7 (CXCR4 and CXCR7) on the neuroblastoma cell surface in vitro and in vivo, causing neuroblastoma cells to migrate to these niches [14-16]. CXCR4 expression is closely correlated with CXCR4 expression in primary neuroblastomas. Bone and bone marrow metastasis, as well as a bad patient performance [17]. IGF-1 and IGF-2, which are formed by neuroblastoma cells, bind to and activate IGF-1R receptors on the surface of preosteoclasts [18]. IGF-1R is expressed on the surface of neuroblastoma cells, and elevated levels of IGF-IR expression in the bone microenvironment cause osteoclast differentiation and the development of osteolytic lesions [19]. Other identified mechanisms of in vitroosteoclast activation and bone degradation include the secreted IL-6 by mesenchymal stem cells, which is activated by neuroblastoma cells [20], and the secreted RANK ligand (RANKL) by pre-osteoclasts [21].

For cells inside the TME, the extracellular matrix (ECM) offers structural support and biochemical interactions. TME cell secretion of numerous ECM macromolecules, such as proteoglycans, polysaccharides, glycosaminoglycans, glycoproteins, and fibrous proteins including collagen and fibronectin, creates and maintains the ECM [22]. These macromolecules serve as a scaffold for tissues, defining their physical properties and affecting cell formation, development, and proliferation [23]. ECM components such as reticulin, collagen fibers, and glycosaminoglycans were shown to be associated with neuroblast, Schwann, and lymphocyte cell behavior in a recent multiparametric study of neuroblastoma TME. To boost the Neuroblastoma Pathology Classification benefit, this review emphasized the importance of incorporating quantitative measurements of TME and ECM characteristics into histopathologic assessments [24]. Neurite outgrowth and suppressed neuroblastoma cell proliferation in vitro in reaction to increasing ECM stiffness [25] show that neuroblastoma cells feel mechanical forces in the ECM and change their gene expression accordingly. It's worth noting that the ECM's mechanical properties influence the expression of N-Myc, a critical oncogenic transcription factor in neuroblastoma [25].

\section{Strategy to model neuroblastoma}

Cancer has historically been studied using twodimensional in vitro models and validated with animal models. Nearly $70 \%$ of biomedical researchers depend on 2D cell models in their preliminary studies. 2D cell culture strategies are commonly used due to being reasonably simple to use, strong in efficacy, and inexpensive. For neuroblastoma studies, a wide array of cell lines has been generated and extensively characterized $[27,28]$. Despite their many advantages, these neuroblastoma cell line models are grown in a monolayer and stripped of the dynamic interactive environment and 3D design, causing altered signaling pathways, cell behavior, development, metabolism, and reaction to stimuli. This 3D tissueengineered systems have seen an uptick in cancer study use as a result (re-viewed in Refs. [30, 31]). both previously explored neuroblastoma models are detailed in this portion (Fig. 2). Regardless of the3D framework studied, the origins of neuroblastoma cells included commercially accessible and widely reported neuroblastoma cell lines [27]. Multicellular tumor spheroid (MCS) is a well-characterized and common scaffold-free 3D cell culture platform [31]. MCS has been documented to be extensively used in drug research and production owing to its capacity to imitate diffusional gradients in vivo and the movement of nutrients, oxygen, and waste [32]. MCSs are generated through the aggregation and compaction of multiple cells in suspension or by successive cell doublings. Forced flotation on non-adhesive structures, the hanging drop process, bioreactor rotational culture, and the force-driven method are all comprehensively reviewed in Ref. [33]. spheroid models allow greater reproducibility, better 
developed cell-type architectures, and adaptability to multi-cell-type co-culturing, which more closely resemble the living tissues. This enhanced physiological importance and sophistication is shown by the increased presence of dermal junctions and desmosomes in tissues in spheroidgrown cells. Despite the benefits of the MCS concept, the model has not yet been broadly accepted in neuroblastoma study. The experiments outlined in this section used this MCS framework to carry out neuroblastoma stem cell testing, drug screening, and the advancement of radiobiology and radio-immunotherapy techniques. Epithelial-to-mesenchymal markers, drug resistance proteins, and survival response regulators were both upregulated in neuroblastoma spheroids grown in 2D culture models, like MCSs of adult cancers [35]. show a core region of necrotic cells surrounded by differentiating cells and by outer-proliferating cells. Growth of cell masses developed in this model influences medication perfusion and sensitivity. Both experiments in Table 1 discovered that the MCS growth trends and cytotoxicity were equivalent to in vivo solid tumor mass, making these models appropriate for novel and repurposing drug screening. The testis sk-n-sh and SH-SY5Y spheroids are more resistant to 15-deoxy-PGJ2 and doxorubicin when developed in three-dimensional media [46]. 10 out of 12 neuroblastoma cell lines showed greater tolerance to cisplatin and $\mathrm{dFdC}$ in $3 \mathrm{D}$ over $2 \mathrm{D}$. It is necessary to consider that this model has a limitation: Not all cell types will shape MCSs or change their life cycle to be able to.

The greatest difficulty in early-phase clinical trials is the portrayal of tumor-specific molecular profile, anatomy, and patient diversity as the number of pediatric patients available for trials is small. In HTS, cancer cell behavior is studied at the processes stage in a biomimetic 3D microenvironment. High Content Screening (HTS) transforms HTS into simultaneous evaluation of morphological modifications, cell viability, and cytotoxicity; along with gene expression research, and apoptosis assays (HCS). MCSs augmented the chemotherapy tolerance of 3D HTSplatforms [35, 41, and 62]." The twelve neuroblastoma cell lines were developed as 3D MCS and screened for cisplatin and gemcitabine (or $\mathrm{dFdC}$ ) cytotoxicity. The research validated the drug susceptibility, synergism, and contribution of the copper absorption and efflux transporters, hCTR1, ATP7A, and ATP7, respectively. Sidarovich et al. used this HCS method to investigate 349 small molecules in five neuroblastoma cell lines [45]. Ponatinib, an FDA-approved multiple tyrosine kinase inhibitor, has shown promise in neuroblastoma treatment utilizing only FDA-approved compounds. To personalize care protocols, high-content drug screening can be used on neuroblastoma cells extracted from patient biopsies, resulting in a cell-based approach to drug assessment. Currently, these models' use in healthcare environments has issues with workforce demands and costliness. additive biomanufacturing, like 3D bio-printing, also offers a cost-effective and userfriendly approach to producing complex 3D structures for cancer research and drug screening, which will enable for the reproduction of the normal shape and function of tumor tissue $[50,53,63]$.

\section{Conclusion}

Cancer development and metastasis are multistage mechanisms that include changes at the genetic, biochemical, tissue, and organ stages, both of which operate through an input and feed-forward mechanism. Following initial investigation in 2D cell cultures, this paradigm has historically been approached using complexin vivom urine models. While these in vivomodels offer a physiologically representative medium for investigating tumors at the organ and organismal stage, they still have significant flaws, such as murine tumor cells, stromal cells, and immune systems. The evolution of 3 Din vitrocancer models over the past 50 years has been motivated by these shortcomings and an increasing awareness of the role of the TME, ECM, and cell structure in disease progression. This shows the potential for significant advancements in the characterization of the neuroblastoma TME as well as the implementation of a well-definedbottom-up disease model. Drug exploration in physiologically applicable and scientifically reproducible 3D tissue-engineered model systems has also made considerable strides, as shown by the studies addressed in this study. This 3Dsystems will speed up the drug research pipeline for personalized therapies and lower the drug production process' attrition rate. These models will also help in the development of novel drug chemistry, formulations, and delivery methods, such as nanoparticle delivery systems. Importantly, 3D cell technology has a significant impact on the number of animals used in preclinical testing, thus addressing the 3Rs ideals of minimizing and removing animals. These 3Din vitrocellmodels can aid in dissecting neuroblastoma biology at the tissue level, even if they are still in the early stages of growth. Using tissue-engineering advancements to deconstruct and re-construct the tumor microenvironment layer by layer in a controllable manner will contribute to a greater understanding of the individual steps leading to neuroblastoma pathogenesis, allowing the disorder to be studied on multiscale and systems-level platforms. Incorporating patient-derived neuroblastoma cells into these 3 -D systems in the future would allow for the testing of susceptibility to new and experimental medications, as well as combined therapies, resulting in a more effective andpersonalizeddesign of preinitiation treatments. In the long term, that would reduce the number of extra chemotherapy rounds provided to pediatric patients. This breakthrough can make it easier to anticipate and identify therapies of high efficacy, which is critical for enhancing the quality of life and, potentially, rising the survival rate of children with neuroblastoma.

\section{References}

[1] R. Patel, A. Kunche, N. Mishra, Z. Bhaiyat, R. Joshi, Comparative review of existing mobile payment systems, International Journal of Applied Engineering Research, 10 (2015), pp. 16873-16884

[2] Shiff NJ, Oen K, Rabbani R, Lix LM. Validation of administrative case ascertainment algorithms for chronic childhood arthritis in Manitoba, Canada. Rheumatol Int. 2017;37(9):1575-1584. doi:10.1007/s00296-017-3734-1

[3] Stuart S, Hickey A, Vitorio R, et al. Eye-tracker algorithms to detect saccades during static and dynamic tasks: a structured review. Physiol Meas. 2019;40(2):02TR01. Published 2019 Feb 26. doi:10.1088/1361-6579/ab02ab

[4] Patel, R., Kunche, A., Mishra, N., Bhaiyat, Z., \& Joshi, P. 
R. (2015). Paytooth-a cashless mobile payment system based on Bluetooth. International Journal of Computer Applications, 120, 3843. https://doi.org/10.5120/21412-4450.

[5] Mahajan V, Venugopal VK, Murugavel M, Mahajan H. The Algorithmic Audit: Working with Vendors to Validate Radiology-AI Algorithms-How We Do It. AcadRadiol. 2020;27(1):132-135. doi:10.1016/j.acra.2019.09.009

[6] Buxton, E. K., Vohra, S., Guo, Y., Fogleman, A., \& Patel, R. (2019). Pediatric population health analysis of southern and central Illinois region: A cross sectional retrospective study using association rule mining and multiple logistic regression. Computer methods and programs in biomedicine, 178, 145-153.

[7] Campbell E. Random Compiler for Fast Hamiltonian Simulation. Phys Rev Lett. 2019;123(7):070503. doi:10.1103/PhysRevLett.123.070503

[8] Devkota S, Aschwanden P, Kunen A, Legendre M, Isaacs KE. CcNav: Understanding Compiler Optimizations in Binary Code. IEEE Trans Vis Comput Graph. 2021;27(2):667-677.doi:10.1109/TVCG.2020.3030357

[9] Rushabh Patel and Rahul Raghvendra Joshi, Envision of IRS (I-Railway System) - based on Cloud Computing, International Journal of Science Engineering and Technology Research (IJSETR), vol. 4, no. 1, January 2015.

[10] Yang X, He H. An advanced compiler designed for a VLIW DSP for sensors-based systems. Sensors (Basel). 2012;12(4):4466-4478. doi:10.3390/s120404466

[11] Rushabh Patel and Rahul Raghvendra Joshi, Introducinga Hi-Tech - Cloud based Public Transport System, International Journal of Computational Intelligence Research ISSN 0973-1873 Volume 11, Number 1 (2015), pp. 19-26

[12] Minkovich K, Srinivasa N, Cruz-Albrecht JM, Cho Y, Nogin A. Programming time-multiplexed reconfigurable hardware using a scalable neuromorphic compiler. IEEE Trans Neural Netw Learn Syst. 2012;23(6):889-901. doi:10.1109/TNNLS.2012.2191795
[13] Rushabh Patel and Rahul Raghvendra Joshi, Evolution \& Envisage of Mobile Network, International Journal of Scientific Engineering and Technology Research ISSN 2319-8885 Vol.03, Issue.01 (January-2014), pp. 01360143.

[14] Lucas J. Vespa et al, A Novel Reinforcement Sample Learning Strategy for Convolution Neural Network in Computer Aided Diagnosis System for Breast Cancer, SIIM 2017 Scientific Session, Posters \& Demonstrations

[15] Lunter G. HMMoC--a compiler for hidden Markov models. Bioinformatics. 2007;23(18):2485-2487. doi:10.1093/bioinformatics/btm350

[16] Rokita JL, Rathi KS, Cardenas MF, et al. Genomic Profiling of Childhood Tumor Patient-Derived Xenograft Models to Enable Rational Clinical Trial Design. Cell Rep. 2019;29(6):1675-1689.e9. doi:10.1016/j.celrep.2019.09.071

[17] Upton K, Modi A, Patel K, et al. Epigenomic profiling of neuroblastoma cell lines.Sci Data. 2020;7(1):116. Published 2020 Apr 14. doi:10.1038/s41597-020-0458-y

[18] Kendsersky NM, Lindsay J, Kolb EA, et al. The B7-H3Targeting Antibody-Drug Conjugate m276-SL-PBD Is Potently Effective Against Pediatric Cancer Preclinical Solid Tumor Models. Clin Cancer Res. 2021;27(10):29382946. doi:10.1158/1078-0432.CCR-20-4221

[19] Raman, Swetha and Buongervino, Samantha N. and Lane, Maria and Zhelev, Doncho V. and Zhu, Zhongyu and Cui, Hong and Martinez, Benjamin and Martinez, Daniel and Wang, Yanping and Upton, Kristen A. and Patel, Khushbu and Rathi, Komal S. and Navia, Carmen T. and Harmon, Daniel B. and Pawel, Bruce and Dimitrov, Dimiter and Maris, John M. and Julien, Jean-Philippe and Bosse, Kristopher, The D3-GPC2-PBD ADC is Potently Efficacious Against Neuroblastoma and SCLC Via Engagement of a Conformational GPC2 Epitope (September 2020). Available at SSRN: https://ssrn.com/abstract=3673604 or http://dx.doi.org /10.2139/ssrn.3673604 